University of Nebraska - Lincoln

DigitalCommons@University of Nebraska - Lincoln

10-19-2002

\title{
Plateau Effects in the Spectra of Electrons Scattered from Atoms in a Strong Laser Field
}

\author{
N. L. Manakov \\ Voronezh State University, manakov@phys.vsu.ru \\ Anthony F. Starace \\ University of Nebraska-Lincoln, astarace1@unl.edu
}

\section{A. V. Flegel}

Voronezh State University, Voronezh, 394693 Russia

M. V. Frolov

Voronezh State University, Voronezh, 394693 Russia

Follow this and additional works at: https://digitalcommons.unl.edu/physicsstarace

Part of the Physics Commons

Manakov, N. L.; Starace, Anthony F.; Flegel, A. V.; and Frolov, M. V., "Plateau Effects in the Spectra of Electrons Scattered from Atoms in a Strong Laser Field" (2002). Anthony F. Starace Publications. 119. https://digitalcommons.unl.edu/physicsstarace/119 


\title{
Plateau Effects in the Spectra of Electrons Scattered from Atoms in a Strong Laser Field
}

\author{
N. L. Manakov1*, A. F. Starace², A. V. Flegel'1, and M. V. Frolov² \\ ${ }^{1}$ Voronezh State University, Voronezh, 394693 Russia \\ *e-mail:manakov@thp.vsu.ru \\ ${ }^{2}$ Department of Physics and Astronomy, University of Nebraska, Lincoln, Nebraska 68588-0111, USA \\ Received July 16, 2002
}

\begin{abstract}
An exact quantum solution of the problem of electron scattering from a short-range potential in the presence of a strong elliptically polarized laser field is obtained. The differential scattering cross section as a function of the number of absorbed (or emitted) photons exhibits a plateau caused by the rescattering of electrons from the scattering center. Numerical results for a linearly polarized laser field are presented, and it is shown that the plateau boundaries agree well with classical estimates. (C) 2002 MAIK "Nauka/Interperiodica".
\end{abstract}

PACS numbers: 32.80.Wr; 34.80.Qb; 03.65.Nk

Among the most interesting and extensively studied nonlinear effects involved in above-threshold ionization (ATI) and high-order harmonic generation (HHG) processes are "plateaus" observed in the ATI and HHG spectra. The presence of a plateau implies that the photoelectron yield resulting from $n$-photon ATI or the intensity of the $n$th pump laser harmonic depend weakly on the number $n$ of absorbed photons over a broad interval (for $n \leqslant n_{\max }$ ). Possessing a single-electron character, these effects have been well studied experimentally and described theoretically, both by a direct numerical solution of the nonstationary (timedependent) Schrödinger equation and within the framework of a quasiclassical approach based on the rescattering concept [1]. According to this concept, a strong oscillating field returns electrons (escaping from atoms due to tunneling ionization events) back to the host ions: rescattering from the ions, the electrons gain additional energy from the pump wave, thus forming a plateau in the ATI spectrum or recombining with the emission of large- $n$ harmonics.

It is interesting to note that the structure of such a plateau depends only weakly on the particular shape of the atomic potential, so that the quasi-classical estimates of the cutoff $\left(n_{\max }\right)$ values and the structures of the HHG and ATI spectra, calculated in the Keldysh approximation (modified to allow for the rescattering effect) [2] within the framework of a zero-radius threedimensional potential model, are in perfect qualitative agreement both with the results of exact numerical calculations and with the experimental data for real atoms (see [3] and references therein). Recently [4], it was demonstrated that the appearance of a plateau related to the rescattering effect is also characteristic of a laserassisted electron-ion recombination process.
A common feature of the aforementioned processes is that an electron in the initial and/or final state is bound to an atom and, hence, is less subject to the action of a laser wave field as compared to electrons in the continuum. This paper presents an example of an exactly solved problem, which shows that plateau effects also accompany free-free electron transitions in a strong laser field (induced multiphoton bremsstrahlung and absorption upon electron scattering from an atom). Therefore, these specific nonlinear effects are inherent in all processes involving the interaction of atomic systems with strong laser fields.

Let us consider the scattering of an electron from a static atomic potential $V(\mathbf{r})$ in the presence of an elliptically polarized laser field with an electric vector $\mathbf{F}(t)=F \operatorname{Re}[\mathbf{e} \exp (-i \omega t)]$, where $\mathbf{e}$ is the unit (complex) vector of polarization: $\mathbf{e} \cdot \mathbf{e}^{*}=1$ and $\mathbf{e} \cdot \mathbf{e}=l$ is the degree of linear polarization of the field $\mathbf{F}(t)(0 \leq l \leq 1)$. In a formalism of the quasi-energy states (QES, see, e.g., [5]), the state of an electron with an asymptotic momentum $\mathbf{p}$ in the potential $V(\mathbf{r})$, corresponding to elastic scattering with the energy $E=p^{2} / 2 m$ in the absence of the field $\mathbf{F}(t)$, is a periodic function of time which satisfies the following equation:

$$
\begin{gathered}
\Phi_{\mathbf{p}}(\mathbf{r}, t)=\chi_{\mathbf{p}}(\mathbf{r}, t)+\Phi_{\mathbf{p}}^{(\mathrm{scatt})}(\mathbf{r}, t)=\chi_{\mathbf{p}}(\mathbf{r}, t) \\
+\int d \mathbf{r}^{\prime} \int d t^{\prime} e^{-i \epsilon\left(t^{\prime}-t\right) / \hbar} G^{(+)}\left(\mathbf{r}, t ; \mathbf{r}^{\prime}, t^{\prime}\right) V\left(\mathbf{r}^{\prime}\right) \Phi_{\mathbf{p}}\left(\mathbf{r}^{\prime}, t^{\prime}\right) .
\end{gathered}
$$

Here, $\chi_{\mathbf{p}}(\mathbf{r}, t)$ and $G^{(+)}\left(\mathbf{r}, t ; \mathbf{r}^{\prime}, t^{\prime}\right)$ are the QES wave function and the retarded Green function of the electron in the field $\mathbf{F}(t)$, respectively; $\epsilon=E+U_{p}$ is the quasienergy; and $U_{p}=e^{2} F^{2} / 4 m \omega^{2}$ is the mean vibrational energy of an electron in the laser field. The scattering 
amplitude is determined by the asymptotic behavior of the scattered wave:

$$
\left.\Phi_{\mathbf{p}}^{\text {(scatt) }}(\mathbf{r}, t)\right|_{r \rightarrow \infty}=\sum_{n>-[E / \hbar \omega]+1}^{\infty} \mathscr{A}_{n} \frac{e^{i p_{n} r / \hbar-i \omega n t}}{r},
$$

where $p_{n}=\sqrt{2 m(E+n \hbar \omega)}$ is the electron momentum in the channel featuring absorption (for $n>0$ ) or emission $(n<0)$ of $|n|$ photons, $\mathscr{A}_{n}$ is the scattering amplitude in the $n$th channel, and the sum over $n$ involves all open channels with $E_{n}=E+n \hbar \omega>0$.

It is possible to solve Eq. (1) exactly when the atomic potential is modeled by a zero-radius potential (ZRP)

$$
V(\mathbf{r})=\frac{2 \pi \hbar^{2}}{\kappa m} \delta(\mathbf{r}) \frac{\partial}{\partial r} r
$$

admitting one weakly bound state $\phi_{E_{0}}(\mathbf{r})$ with an energy of $E_{0}=-\hbar^{2} \kappa^{2} / 2 m$. It should be noted that the use of the ZRP in this problem is much better justified as compared to the cases of ATI or HHG because of the short-range character of the potential of a neutral atom. Below we use dimensionless quantities, whereby the energies (including $U_{p}$ ) are measured in units of $\left|E_{0}\right|$; frequencies, in units of $\left|E_{0}\right| / \hbar$; and the field amplitudes $F$, in units of $F_{0}=\sqrt{2 m\left|E_{0}\right|^{3}} /|e| \hbar$. For example, the scattering on a hydrogen atom is characterized by $\left|E_{0}\right|=$ $0.754 \mathrm{eV}=0.0277$ a.u. (the binding energy of $\mathrm{H}^{-}$) and $F_{0}=3.362 \times 10^{7} \mathrm{~V} / \mathrm{cm}=6.52 \times 10^{-3}$ a.u.

In the ZRP model, the problem reduces to calculation of the Fourier coefficients $f_{k}$ determining behavior of the scattering wave function $\Phi_{\mathbf{p}}(\mathbf{r}, t)$ at $\mathbf{r} \longrightarrow 0$ :

$$
\left.\Phi_{\mathbf{p}}(\mathbf{r}, t)\right|_{\mathbf{r} \rightarrow 0}=\left(\frac{1}{r}-1\right) f(t)=\left(\frac{1}{r}-1\right) \sum_{k} f_{k} e^{i k \omega t} .
$$

Indeed, using the well-known expression for $G^{(+)}\left(\mathbf{r}, t ; 0, t^{\prime}\right)$, the amplitude $\mathscr{A}_{n}$ in Eq. (2) can be represented as (see the results obtained in [6] for a circularly polarized field $\mathbf{F}(t))$

$$
\begin{gathered}
\mathscr{A}_{n}=\sum_{m, s} i^{m} e^{i m \tilde{\phi}_{n}} f_{2 s-n-m} J_{s}\left(l \frac{U_{p}}{2 \omega}\right) J_{m}\left(\frac{2 F}{\omega^{2}}\left|\mathbf{e} \cdot \mathbf{p}_{n}\right|\right), \\
e^{i \tilde{\phi}_{n}}=\frac{\mathbf{e} \cdot \mathbf{p}_{n}}{\left|\mathbf{e} \cdot \mathbf{p}_{n}\right|},
\end{gathered}
$$

and the differential scattering cross section is

$$
\frac{d \sigma_{n}}{d \Omega}=\frac{p_{n}}{p}\left|\mathscr{A}_{n}\right|^{2} \text {. }
$$

A system of linear inhomogeneous equations for $f_{k}$ follows from Eq. (1) with boundary condition (4) (see the analogous calculations in [7] for ionization from the state $\phi_{E_{0}}(\mathbf{r})$ in the ZRP):

$$
\begin{aligned}
& \left(1+i \sqrt{\mathscr{E}_{n, \delta}}+M_{n, n}\left(\mathscr{E}_{0, \delta}\right)\right) f_{2 n+\delta} \\
& +\sum_{m \neq n} M_{n, m}\left(\mathscr{E}_{0, \delta}\right) f_{2 m+\delta}=c_{2 n+\delta},
\end{aligned}
$$

where

$$
\begin{gathered}
c_{k}=-i^{k} \sum_{s} e^{i(2 s+k) \tilde{\phi}} J_{s}\left(l \frac{U_{p}}{2 \omega}\right) J_{k+2 s}\left(\frac{2 F}{\omega^{2}}|\mathbf{e} \cdot \mathbf{p}|\right), \\
M_{n, m}\left(\mathscr{E}_{0, \delta}\right)=\frac{i^{(m-n)}}{\sqrt{4 \pi i}} \int_{0}^{\infty} \frac{d \tau}{\tau^{3 / 2}} e^{i\left(\mathscr{E}_{0, \delta}-(n+m) \omega\right) \tau} \\
\times\left\{e^{i z} J_{n-m}\left(l z-l\left(U_{p} / \omega\right) \sin \omega \tau\right)-\delta_{n, m}\right\}, \\
z=\frac{4 U_{p}}{\omega^{2} \tau} \sin ^{2} \frac{\omega \tau}{2}, \quad e^{i \tilde{\phi}}=\frac{\mathbf{e} \cdot \mathbf{p}}{|\mathbf{e} \cdot \mathbf{p}|},
\end{gathered}
$$

$\mathscr{E}_{n, \delta}=p^{2}-(2 n+\delta) \omega$, and $\delta=0(1)$ for even (odd) $k$ values. Note that the coefficients $f_{k}$ with even and odd numbers are determined independently (for ionization, the incident wave $\chi_{\mathbf{p}}(\mathbf{r}, t)$ in Eq. (1) is absent and the boundary condition (4) contains only the coefficients $f_{k}$ with even $k$ [7]). Numerical values of $f_{k}$ are obtained by solving a system of equations (7) "truncated" at $n$; the rate of convergence with respect to $n$ (the number of coefficients taken into account) depends on $F, \omega$, and $p$.

The exact relations (5)-(8) admit analytical treatment in some limiting cases. In particular, ignoring nondiagonal matrix elements $M_{n, m}$ in relations (7), we obtain

$$
f_{2 n+\delta}^{(0)}=\frac{c_{2 n+\delta}}{1+M_{n, n}\left(\mathscr{E}_{0, \delta}\right)+i \sqrt{\mathscr{E}_{n, \delta}}} .
$$

Note that, in a weak field (i.e., for $F^{2} \ll \omega^{3}$ or $U_{p}=$ $F^{2} / 2 \omega^{2} \ll \omega$, we have $\left.M_{m, n} \sim F^{2\left(|m-n|+\delta_{m, n}\right)}\right)$. For a circularly polarized field $\mathbf{F}(t)(l=0)$, nondiagonal matrix elements are zero and the approximation (9) coincides with the exact expression for $f_{k}$; substituting this expression into (5) yields the well-known result for a circularly polarized field [6]. For $0<l \leq 1$, the poles of the coefficients $f_{k}^{(0)}$ in the complex plane $E$ (i.e., the zeros of the denominator in (9)) give an equation for the complex quasi-energy. The imaginary part of the quasienergy coincides with the width of level $\phi_{E_{0}}(\mathbf{r})$ in the Keldysh approximation of multiphoton ionization theory [7].

With neglect of the term $M_{n, n}$ in expression (9), we can obtain simple closed relations for $d \sigma_{n} / d \Omega$ in the Born and/or low-frequency limits, whereby the term 


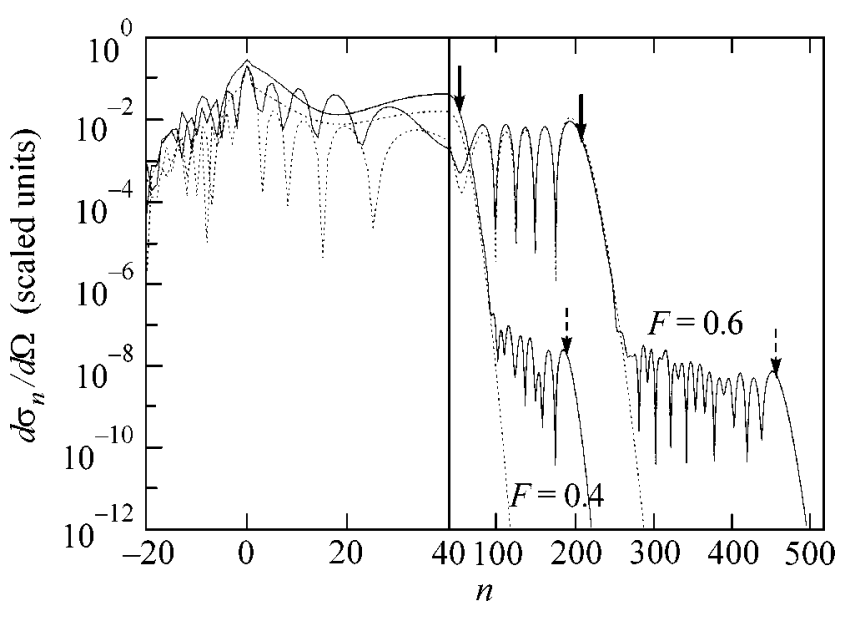

Fig. 1. The differential cross section (in dimensionless units, see the text) of forward scattering $(\theta=0)$ as a function of the number of absorbed (emitted) photons calculated for $\omega=0.155, E=20.5 \omega=3.1775$, and $F=0.4\left(U_{p}=3.33=\right.$ $21.48 \omega)$ and $0.6\left(U_{p}=7.49=48.34 \omega\right)$ using the exact equations (solid curves) and the approximate formula (11) (dashed curves). Solid and dashed arrows indicate the Kand R-plateau cutoffs estimated by formulas (15) and (19), respectively.

$(2 n+\delta) \omega$ in the relation $\sqrt{\mathscr{E}_{n, \delta}}=\sqrt{p^{2}-(2 n+\delta) \omega}$ can be omitted. For example, in the low-frequency limit,

$$
f_{k}^{(\mathrm{KW})}=\frac{c_{k}}{1+i p},
$$

where $(1+i p)^{-1}$ is the exact amplitude of elastic scattering in the ZRP model. Substituting (10) into (5) and taking into account orthogonality of the Bessel functions,

$$
\sum_{k=-\infty}^{\infty} J_{v+k}(u) J_{k}(u)=\delta_{v, 0}
$$

we arrive at

$$
\frac{d \sigma_{n}^{K W}}{d \Omega}=\frac{p_{n}}{p\left(1+p^{2}\right)} J_{n}^{2}\left(\frac{2 F}{\omega^{2}}\left|\mathbf{e} \cdot\left(\mathbf{p}-\mathbf{p}_{n}\right)\right|\right),
$$

which is the Kroll-Watson result [8]. In the case of fast electrons ( $p \gg 1$, or $p \gg \sqrt{2 m\left|E_{0}\right|}$ in absolute units), we can omit unity in the denominators of (10) and (11), thus obtaining the Bunkin-Fedorov result for the ZRP model [9].

An analysis shows that the main difference of the results $[8,9]$ from the exact solution is due to neglect of the nondiagonal matrix elements $M_{n, m}$ in expression (9). These elements describe high-order effects (i.e., rescattering effects) of interaction with the atomic potential. In the lowest order, allowance for the rescat- tering corresponds to an iteration of the system (7) based on the zero approximation (9):

$$
f_{2 n+\delta}^{(1)}=\frac{c_{2 n+\delta}-\sum_{m \neq n} M_{n, m}\left(\mathscr{E}_{0, \delta}\right) f_{2 m+\delta}^{(0)}}{1+M_{n, n}\left(\mathscr{E}_{0, \delta}\right)+i \sqrt{\mathscr{E}_{n, \delta}}} .
$$

In the ZRP model, approximation (12) is equivalent to taking into account the first correction of the atomic potential to the ATI amplitude in the Keldysh approximation. The results of numerical calculations (see below) show that, for frequencies $\omega<1$, approximation (12) ensures a high accuracy (the difference from the exact results being below 5\%) in a broad range of field intensities.

Since the rescattering effects are most significant for a linear polarization of the field $\mathbf{F}(t)$, the numerical results will be presented for the simplest geometry, in which the initial electron momentum is directed along the linear polarization axis and the angular distribution of scattered electrons depends only on the angle $\theta$ between $\mathbf{p}$ and $\mathbf{p}_{n}$. Figure 1 shows the differential forward scattering cross sections $(\theta=0)$ calculated, by approximate formula (11) and by exact formulas (5)(8), as a function of the number of absorbed (emitted) photons for $\omega=0.155$; the initial electron energy $E=$ $20.5 \omega$, and two values of the field amplitude, $F=0.4$ and 0.6. For scattering from a hydrogen atom, these parameters correspond to a $\mathrm{CO}_{2}$ laser frequency, the initial electron energy $E=2.4 \mathrm{eV}$, and the field intensities $I \approx 2.4 \times 10^{11}$ and $5.4 \times 10^{11} \mathrm{~W} / \mathrm{cm}^{2}$ (in such fields, the probability of tunneling ionization of hydrogen is negligibly small and the effect of ionization of target atoms in the course of scattering can be ignored).

A clearly pronounced feature in Fig. 1 is the presence of two plateaus in the differential scattering cross section as a function of the number of absorbed photons. The first plateau (corresponding to smaller $n$ ) is obtained for both exact and approximate calculations and is five-to-six orders of magnitude higher than the second plateau. A fully analogous situation takes place in the ATI spectra (see, e.g., [3]), where the first plateau is well described in the Keldysh approximation (and is referred to below as the K-plateau), while the second is due to the rescattering effects (R-plateau). The results of a numerical analysis show that the lengths of both the $\mathrm{K}$ - and R-plateaus for electron scattering at fixed $E$ and $\omega$ increase with $F$, while $n_{\max }^{R}$ increases much faster than does $n_{\max }^{K}$. On the contrary, as the electron energy $E$ increases, the length (in the $n$ scale) of both plateaus decreases, the K-plateau contracting much faster than the R-plateau. For example, at $F=0.4$ and $E=30.5 \omega$ $=1.42 U_{p}$, the cross section smoothly decays with increasing $n$ unless the R-plateau appears (i.e., the 


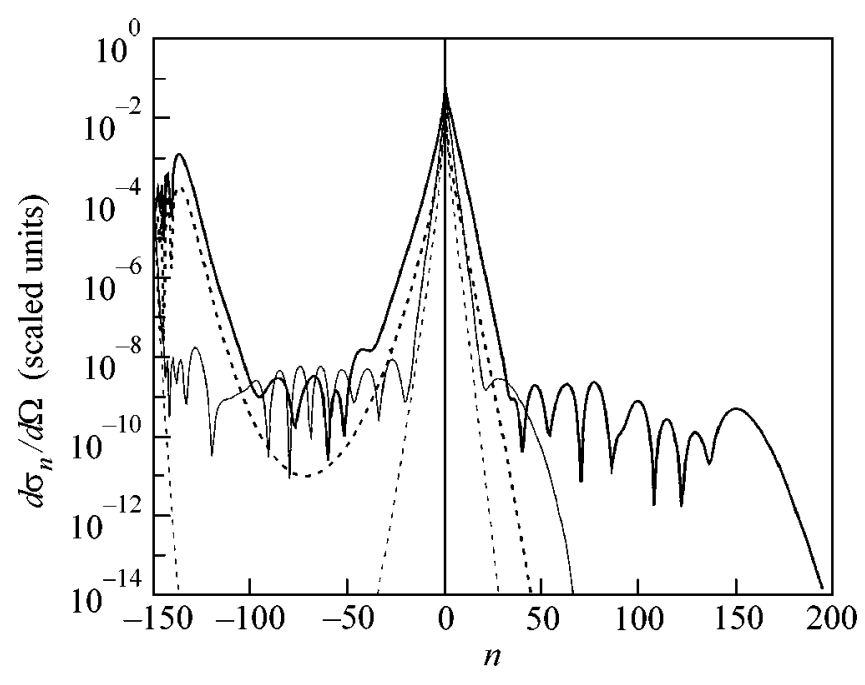

Fig. 2. The same as in Fig. 1, but for $E=150.5 \omega=23.3275$. Thin and thick solid curves refer to $F=0.4\left(U_{p}=3.33=\right.$ $21.48 \omega)$ and $0.5\left(U_{p}=5.2=33.57 \omega\right)$, respectively.

K-plateau is virtually absent, while the R-plateau still has a length of $n_{\max }^{R} \approx 100$ ).

Figure 2 shows the plots of $d \sigma_{n} / d \Omega$ versus $n$ calculated for the energy $E=150.5 \omega$ and $F=0.4$ and 0.5 $\left(U_{p}=21.48 \omega\right.$ and $33.57 \omega$, respectively). For these parameters, the K-plateau is completely absent and the R-plateau appears only in a sufficiently strong field $(F \geq 0.5)$. Another characteristic feature in the highenergy cross section is the appearance of the R-plateau in the process of induced emission $(n<0)$ in a broad range of $n$ (for which the Kroll-Watson and BunkinFedorov approximations give a deep minimum and differ significantly from the exact results).

As the scattering angle $\theta$ increases, the structure of the spectrum of scattered electrons significantly changes because the role of rescattering effects decreases. Evolution of the plateau structure is illustrated in Fig. 3. As was noted above for $\theta=0$, the $\mathrm{K}$-plateau at the $E, F$, and $\omega$ values indicated in Fig. 3 is absent. As the $\theta$ value increases, the R-plateau length decreases, while the K-plateau becomes clearly manifested at $\theta \sim \pi / 3$, significantly masks the R-plateau at $\theta>\pi / 2$, and completely determines the spectrum of scattered electrons for still greater angles of scattering. An analogous situation is also observed for other values of the parameters, so that the scattering by large angles is well described by formula (11). However, it should be pointed out that the interval of small angles $\Delta \theta$, in which approximation (11) is inapplicable (i.e., the region of "critical geometry," where the momentum transfer $\mathbf{p}-\mathbf{p}_{n}$ is perpendicular to the field polarization plane and the argument of the Bessel function in (11) is close to zero), significantly expands with increasing $n$ and may reach up to $\Delta \theta \sim \pi / 2$.

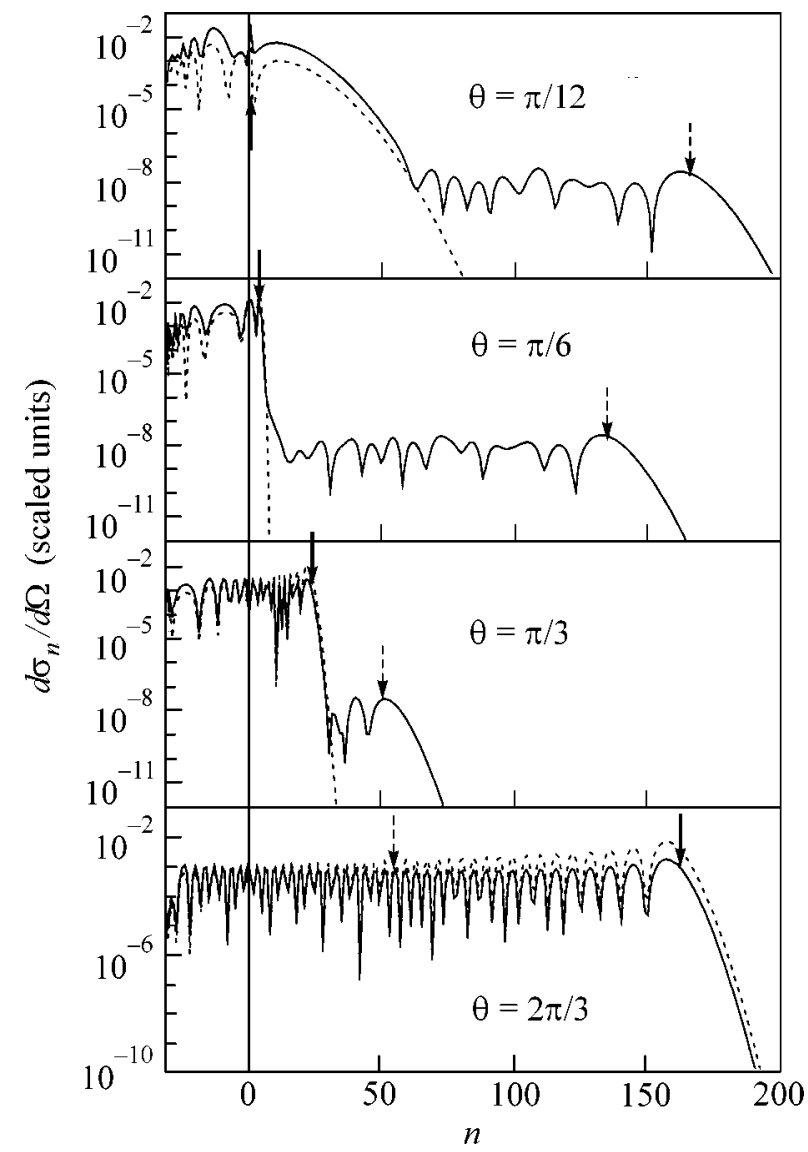

Fig. 3. The plots of $d \sigma_{n} / d \Omega$ versus $n$ for various scattering angles $\theta$ calculated for $F=0.4, \omega=0.155$, and $E=30.5 \omega$ using the exact equations (solid curves) and approximate formula (11) (dashed curves). Solid and dashed arrows indicate the K- and R-plateau cutoffs according to quasi-classical estimates (see the text).

The above results admit a simple qualitative interpretation within the framework of a quasi-classical approach, by analogy with interpretation of the plateau effects in HHG [2], ATI [10], and electron-ion recombination spectra [4]. The classical equations employed in such an analysis represent the equations for saddle points in the quasi-classical calculation of integrals in the amplitude of the process under consideration (see, e.g., [11]). Restricting our consideration to the case of a one-dimensional motion of the electron along the direction of linear polarization of the field $\mathbf{F}(t)$ (forward scattering and backscattering), the K-plateau cutoff $\left(n_{\max }^{K}\right)$ can be estimated using an equation expressing the law of conservation of the kinetic energy of the electron in the field upon collision with a scattering center at a time $t$ :

$$
\left[p+\frac{1}{c} A(t)\right]^{2}=\left[k(t)+\frac{1}{c} A(t)\right]^{2}
$$


where $A(t)=-(c F / \omega) \sin \omega t$ is the vector potential, $p$ is the initial momentum, and $k(t)$ is the momentum upon collision. Using a solution to Eq. (13),

$$
k(t)=-p-2 \frac{A(t)}{c},
$$

it is possible to calculate the maximum energy gained by the electron as a result of interaction with the scattering center:

$$
n_{\max }^{K} \omega \equiv \max \left(k(t)^{2}-p^{2}\right)=8 U_{p} \mp 4 \sqrt{2 U_{p} E},
$$

where the minus and plus signs correspond to forward scattering and backscattering, respectively. Thus, for forward scattering, the K-plateau in the bremsstrahlung absorption spectrum disappears at $E \geq 2 U_{p}$. It should be noted that result (15) can also be obtained more formally, by equating the argument and index of the Bessel function (11), which corresponds to the region of transition from oscillating to decaying behavior of $J_{n}(x)$. As can be seen from the results of numerical calculations presented in Figs. 1-3, estimate (15) agrees well with the exact quantum-mechanical calculation. The difference in length of the K-plateau for the backscattering and forward scattering is proportional to $p F / \omega^{2}$ and becomes large in a strong low-frequency field, which explains why backscattering predominates upon absorption of a large number of photons.

The R-plateau cutoff ( $n_{\max }^{R}$ ) can be estimated within the framework of the rescattering concept, by considering first the collision of an electron with a scattering center at a time instant $t$, then the motion of the electron in the wave field (over the time interval from $t$ to $t+\tau$ ) and finally, the repeated scattering (rescattering) at a time instant $t+\tau$. This three-step process is described by the following system of equations:

$$
\begin{gathered}
{\left[p+\frac{1}{c} A(t)\right]^{2}=\left[\tilde{k}(t)+\frac{1}{c} A(t)\right]^{2},} \\
\int_{t}^{t+\tau}\left[\tilde{k}\left(t^{\prime}\right)+\frac{1}{c} A\left(t^{\prime}\right)\right] d t^{\prime}=0, \\
{\left[\tilde{k}(t+\tau)+\frac{1}{c} A(t+\tau)\right]^{2}=\left[k(t+\tau)+\frac{1}{c} A(t+\tau)\right]^{2},}
\end{gathered}
$$

where Eqs. (16) and (18) are analogous to Eq. (13) and express the conservation of the kinetic energy of the electron upon collisions at the time instants $t$ and $t+\tau$, while Eq. (17) describes the return of the electron to the scattering center at the time instant of rescattering $(t+\tau)$. The joint solution of Eqs. (16)- (18) leads to the following expression for a maximum value of the elec- tron energy $\left(n_{\max }^{R} \omega \equiv \max \left(k(t+\tau)^{2}-p^{2}\right)\right)$ gained in the course of the "double" scattering:

$$
n_{\max }^{R} \omega=8 U_{p} K-E, \quad K=\max \left(g_{ \pm}^{2}(\tau)\right),
$$

where

$$
\begin{gathered}
g_{ \pm}(\tau)=\frac{1}{\zeta^{2}+s^{2}}\left[a\left(\zeta^{2}-s^{2}\right) \pm 2 \zeta s \sqrt{\zeta^{2}+s^{2}-a^{2}}\right] \\
a=\frac{p \omega}{2 F}, \quad \zeta=\frac{s}{\omega \tau}-\cos \frac{\omega \tau}{2}, \quad s=\sin \frac{\omega \tau}{2} .
\end{gathered}
$$

Here, the functions $g_{+}(\tau)$ and $g_{-}(\tau)$ correspond to the forward scattering and backscattering, respectively. The coefficient $K$ tends to a maximum $\left(K_{\max }=1.25\right)$ for $a \longrightarrow 0$, which corresponds to small primary electron energies and/or superstrong low-frequency fields. In this case, the maximum energy gained by the electron in the field amounts to $10 U_{p}$ (the same maximum energy is gained by the high-energy photoelectrons in the ATI process [10]). In the opposite case, $a \geq 1$ (or $\left.E \gtrsim 8 U_{p}\right)$, the coefficient $K$ is small $(K \lesssim 1)$ and rapidly decays with increasing $a$. Note that the classical equations possess no real solutions for $E>10 U_{p}$ (the functions $g_{ \pm}(\tau)$ are complex), which corresponds to the absence of rescattering effects: electrons cannot return to the scattering center (see [4] for the electron-ion recombination and [10] for ATI). In the case of backscattering $(\theta=\pi)$ of electrons with the energies $E \gtrsim$ $0.1 U_{p}, n_{\max }^{K}>n_{\max }^{R}$, the less "intense" R-plateau related to the rescattering is masked by the K-plateau corresponding to a single (direct) scattering (Fig. 3).

The above results show that a correct allowance for the atomic potential essentially determines the pattern of electron scattering from an atom in a strong laser field and accounts for the appearance of plateaus in the spectra of electrons scattered by small angles. The plateau effects observed in induced bremsstrahlung processes are of the same nature as those in other interactions of atoms with intense laser fields.

This study was supported jointly by the U.S. Civilian Research and Development Foundation and the Ministry of Education of the Russian Federation (grant no. VZ-010-0), by the Russian Foundation for Basic Research (project no. 00-02-17843), Center for Competitions of the Ministry of Education of the Russian Federation (project no. E00-3.2-515), and the U.S. National Science Foundation (grant no. PHY0070980).

\section{REFERENCES}

1. M. Yu. Kuchiev, Pis'ma Zh. Éksp. Teor. Fiz. 45, 319 (1987) [JETP Lett. 45, 404 (1987)]; K. J. Schafer, B. Yang, L. F. DiMauro, and K. C. Kulander, Phys. Rev. Lett. 70, 1599 (1993); P. B. Corkum, Phys. Rev. Lett. 71, 1994 (1993). 
2. L. V. Keldysh, Zh. Éksp. Teor. Fiz. 47, 1945 (1964) [Sov. Phys. JETP 20, 1307 (1964)].

3. P. Salières, B. Garrè, L. LeDèroff, et al., Science 292 , 902 (2001).

4. D. B. Milošević and F. Ehlotzky, Phys. Rev. A 65, 042504 (2002).

5. N. L. Manakov, V. D. Ovsiannikov, and L. P. Rapoport, Phys. Rep. 141, 319 (1986).

6. I. J. Berson, J. Phys. B 8, 3078 (1975).

7. N. L. Manakov and A. G. Faĭnshteĭn, Zh. Éksp. Teor. Fiz. 79, 751 (1980) [Sov. Phys. JETP 52, 382 (1980)].
8. N. M. Kroll and K. M. Watson, Phys. Rev. A 8, 804 (1973).

9. F. V. Bunkin and M. V. Fedorov, Zh. Éksp. Teor. Fiz. 49, 1215 (1965) [Sov. Phys. JETP 22, 844 (1965)].

10. G. G. Paulus, W. Becker, W. Nicklich, and H. Walther, J. Phys. B 27, L703 (1994).

11. M. Lewenstein, K. C. Kulander, K. J. Schafer, and P. H. Bucksbaum, Phys. Rev. A 51, 1495 (1995).

Translated by P. Pozdeev 